\title{
A convenient method to generate and maintain poly(A)-encoding DNA sequences required for in vitro transcription of mRNA
}

\author{
Patrick Arbuthnot, ${ }^{\star 1}$, Abdullah Ely' \& Kristie Bloom ${ }^{1}$
}

\begin{abstract}
Generating mRNA in vitro to encode therapeutic or cell-modifying proteins is rapidly gaining favor. An important factor that determines efficiency of translation from in vitro transcribed mRNA is the length of the $3^{\prime} \operatorname{poly}(A)$ sequence. However, reproducibly generating and maintaining templates from circular plasmids to have consistent lengths of the homo poly(A) sequences is challenging. The procedure reported here entails repeated restriction digestion with type IIS enzymes, ligation and circular plasmid propagation. The homopolymeric sequence of approximately $100 \mathrm{bp}$ that is generated using the method is approximately equal to the number of $3^{\prime}$ A residues found in the mRNA of mammalian cells. Evaluating expression in vivo of a reporter transcript produced using this method showed efficient expression in vivo.
\end{abstract}

\section{METHODS SUMMARY}

A method that uses type IIS restriction enzymes to build a poly(A:T) tract of an mRNA-encoding sequence within a circular plasmid is described. Repeated asymmetric cleavage followed by ligation enables lengthening of the homo tract to an approximate maximum of 100 poly(A:T) base pairs. mRNA transcribed from this template is efficiently translated in vivo.

\section{KEYWORDS:}

in vitro transcription $\cdot \mathrm{mRNA} \cdot$ plasmid - $\operatorname{poly}(A) \cdot$ type IIS restriction enzymes

'Wits/SAMRC Antiviral Gene Therapy Research Unit, School of Pathology, Faculty of Health Sciences, University of the Witwatersrand, Private Bag 3, WITS 2050, Johannesburg, South Africa; *Author for correspondence: Patrick.Arbuthnot@wits. ac.za

BioTechniques 66: 37-39 (January 2019) 10.2144/btn-2018-0120
Use of mRNA to encode therapeutic, cellmodifying or immunogenic proteins has recently gained popularity [1]. Valuable features of mRNA for such application are: 1) a cytoplasmic site of action, unlike DNA, which simplifies delivery when formulated in synthetic nonviral vectors; 2) RNA is shortlived in vivo to make control of dose easier to achieve; and 3) mRNA does not recombine with DNA, and resultant mutation of the host genome is avoided.

For large-scale preparation of mRNAs in vitro, sequences are typically generated using T7 RNA polymerase. Products of the transcription reaction usually have a $5^{\prime}$ CAP or internal ribosomal entry site (IRES), 5' untranslated region (5'UTR), proteincoding open reading frame (ORF), $3^{\prime} U T R$ and poly $(A)$ sequences at the $3^{\prime}$ end. The number of A residues at the $3^{\prime}$ end of mRNA is variable and has been reported to range between 67 to $96 \mathrm{nt}$ in actively translated mRNAs of mammalian cells [2]. Ensuring an optimal length of the poly $(A)$ tail is vital for efficient translation and shortening of these sequences leads to decay of mRNA (reviewed in $[3,4]$ ). Multiple A residues may be added to synthetic RNA using a poly(A) polymerase from Escherichia coli (EPAP) [5] or during transcription from an encoding template $[6,7]$. When using EPAP to add $A$ residues to the $3^{\prime}$ end of mRNA, achieving consistency of the tail lengths may be difficult $[6,7]$. A complication of using poly(A:T)-containing circular plasmids is that the homopolymeric A:T base pairs in these vectors are unstable and prone to shortening during replication in bacteria $[6,8]$. Use of a linear plasmid vector was recently employed to increase stability of longer poly(A:T) tracts [6].

To improve on generating poly $(A)$ encoding templates from widely used circular plasmid DNA, we developed template DNA that may be lengthened using standard procedures of restriction digestion with ligation and propagation in bacteria. The starting DNA used to engineer the plasmid DNA sequences is depicted in Figure 1A. An mRNA-generating plasmid with 5'UTR comprising the entire IRES of hepatitis $\mathrm{C}$ virus (HCV) [9] and 3'UTR derived from human $\beta$-globin was generated in house. Insertion of a gene of interest, which was Firefly Luciferase (FLuc) in the example used here, was achieved using Gibson assembly [10]. The sequence of the poly(A:T)-encoding region comprised 13 A:T base pairs with flanking Faql and $B v e l$ type IIS restriction sites. The Bvel site was positioned at the $5^{\prime}$ end and the Faq site at the $3^{\prime}$ end of the poly(A:T)-encoding sequence. These type IIS restriction enzymes have unique cleavage sites in the poly $(A: T)$ flanking regions. Orientation of the recognition sites was such that the enzymes each cleaved asymmetrically within the poly(A:T) sequence to generate complementary sticky ends comprising four T or A overhangs. In addition, a Sapl restriction site was placed immediately $3^{\prime}$ of the poly(A:T) tracts, and this enzyme was used to linearize the plasmid to generate a template for in vitro transcription. A Sapl site that naturally occurs within the FLuc ORF was abolished using site-directed mutagenesis while retaining the amino acid sequence of the reporter enzyme. The first step of the process to lengthen the A:T sequence entailed two independent double digestions of the plasmid (Figure 1B). After cleavage with Bvel and Scal or Faql and Scal according to procedures recommended by the supplier of the enzymes (New England Biolabs, MA, USA), fragments that each contained the longer poly(A:T) sequences were purified. A replicative plasmid was reconstituted after ligation (T4 DNA ligase, ThermoFisher, MA, USA) and propagated in chemically competent bacteria of the XL1-Blue strain (Agilent Technologies, CA, USA). Scal has a unique recognition site 


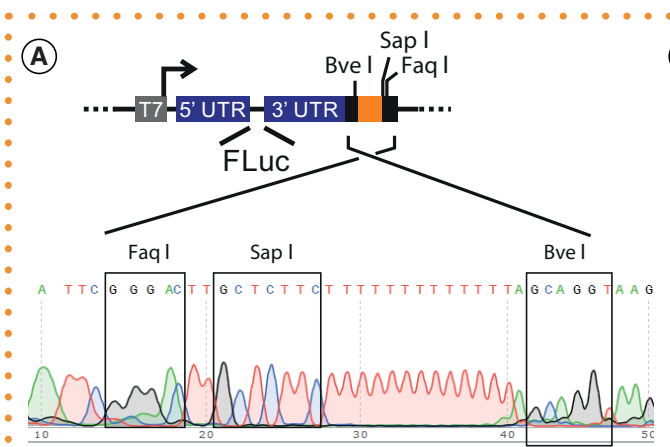

(C)

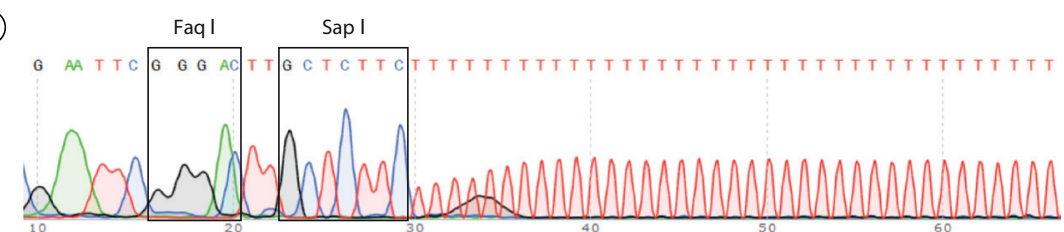

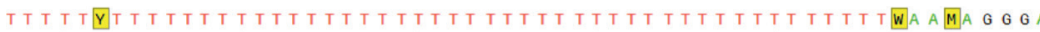

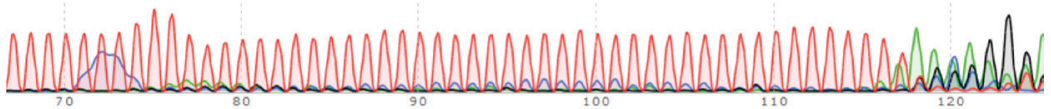

(D)

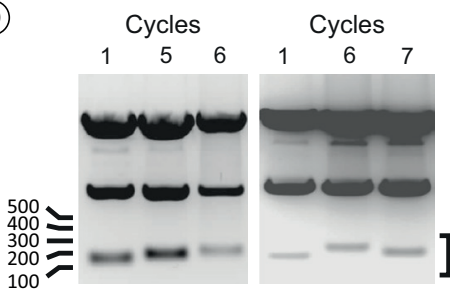

(B)

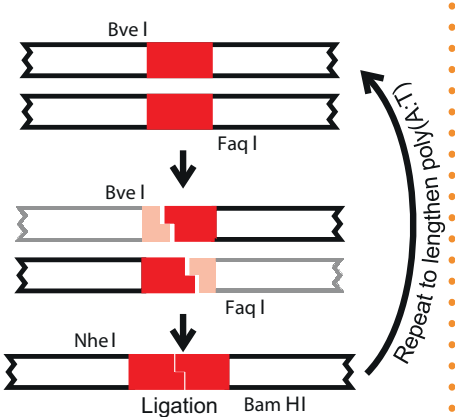

therefore estimated from the length of restriction fragments that encompassed the region of interest (Figure 1D). Our observations indicated a maximum length of the poly(A:T) tract of 80 to $100 \mathrm{bp}$ was achieved after six cycles of the repeated cloning process. This is similar to naturally occurring poly(A) in mRNA and should be suitable for intracellular translation [2]. Information available online indicates that this length of the poly (A)encoding sequences also exceeds what can typically be guaranteed by commercial suppliers of synthetic duplex DNA. Linear templates derived from intermediates of the sequential steps of the cloning reactions, which lacked FLuc sequences, were used to generate mRNA using T7 RNA polymerase (TranscriptAid T7 High Yield Transcription Kit, ThermoFisher). Electrophoretic resolution of the mRNA confirmed increasing lengths of the transcripts, which correlated with the lengthening poly $(A)$ containing $3^{\prime}$ ends of the mRNA (Figure 1E).

To evaluate whether the poly (A)containing mRNA sequences were functional, FLuc-encoding plasmid DNA was also linearized with Sapl and transcribed in vitro using T7 RNA polymerase. Assessment of translation from the template in vivo was determined using an institutionally approved protocol (AESC 2018/21/5C) for transfection of murine hepatocytes using hydrodynamic injection [11]. Expression of FLuc in the liver was measured using standard bioluminescence imaging with an IVIS Kinetic Bioluminescence imager (PerkinElmer, MA, USA). Representative data obtained $3 \mathrm{~h}$ after administration of $5 \mu \mathrm{g}$ of mRNA are shown in Figure 1F. After an exposure of $60 \mathrm{~s}, 1.045 \times 10^{7}$ photons were detected in the region of interest over the liver of the mouse shown in Figure 1F. This value is comparable to that observed after similar administration of commercially available FLuc-encoding mRNA (Trilink, CA, USA). These data verify that the poly(A) tail of the in vitro transcribed transcript was functional in vivo.

The simple method described here is convenient for propagation of plasmids with transcripts that may be used to serve as templates for preparation of efficiently translated mRNA. Moreover, should shortening of the sequence occur, reconstitution 
of the length of the poly $(A)$ tract could be conveniently achieved.

\section{AUTHOR CONTRIBUTIONS}

PA conceived the idea, carried out most of the DNA manipulation and in vitro transcription with contributions from KB. $A E$ performed the hydrodynamic injection and bioluminescence analysis. The manuscript was initially drafted by PA and finalized with input from $A E$ and $K B$.

\section{FINANCIAL \& COMPETING INTERESTS DISCLOSURE}

Financial assistance of the authors' laboratory, which was received from the South African Medical Research Council, National Research Foundation, Johnson \& Johnson Innovation and the Poliomyelitis Research Foundation, is gratefully acknowledged. The authors have no other relevant affiliations or financial involvement with any organization or entity with a financial interest in or financial conflict with the subject matter or materials discussed in the manuscript apart from those disclosed.

No writing assistance was utilized in the production of this manuscript.

\section{OPEN ACCESS}

This work is licensed under the AttributionNonCommercial-NoDerivatives 4.0 Unported License. To view a copy of this license, visit http://creativecommons.org/ licenses/by-nc-nd/4.0/

\section{REFERENCES}

1. Sahin U, Kariko K, Tureci O. mRNA-based therapeutics - developing a new class of drugs. Nat. Rev. Drug Discov. 13(10), 759-780 (2014)

2. Subtelny AO, Eichhorn SW, Chen GR, Sive H, Bartel DP. Poly(A)-tail profiling reveals an embryonic switch in translational control. Nature 508(7494), 66-71 (2014).

3. Chen $\mathrm{CY}$, Shyu AB. Mechanisms of deadenylation-dependent decay. Wiley Interdiscip. Rev. RNA 2(2), 167-183 (2011).

4. Jalkanen AL, Coleman SJ, Wilusz J. Determinants and implications of mRNA poly(A) tail size - does this protein make my tail look big? Semin. Cell Dev. Biol. 34 24-32 (2014).

5. Cao GJ, Sarkar N. Identification of the gene for an Escherichia coli poly(A) polymerase. Proc. Natl Acad. Sci. USA 89(21), 10380-10384 (1992).

6. Grier $A E$, Burleigh $S$, Sahni J et al. pEVL: a linear plasmid for generating mRNA IVT templates with extended encoded Poly(A) sequences. Mol. Ther. Nucleic Acids 5 e306 (2016)

7. Holtkamp S, Kreiter S, Selmi A et al. Modification of antigen-encoding RNA increases stability, translational efficacy, and T-cell stimulatory capacity of dendritic cells. Blood 108(13), 4009-4017 (2006).

8. Tautz D, Schlotterer C. Simple sequences. Curr. Opin. Genet. Dev. 4(6), 832-837 (1994)

9. Takamizawa A, Mori C, Fuke I et al. Structure and organization of the hepatitis $\mathrm{C}$ virus genome isolated from human carriers. J. Virol. 65(3), 1105-1113 (1991).

10. Gibson DG, Young L, Chuang RY, Venter JC, Hutchison CA 3rd, Smith HO. Enzymatic assembly of DNA molecules up to several hundred kilobases. Nat. Methods 6(5), 343-345 (2009)

11. Yang PL, Althage A, Chung J, Chisari FV. Hydrodynamic injection of viral DNA: a mouse model of acute hepatitis $B$ virus infection. Proc. Natl Acad. Sci. USA 99(21), 13825-13830 (2002)

\section{OLYMPUS}

Your Vision, Our Future

\section{Discover the Possibilities}

\section{with the FLUOVIEW ${ }^{\circledR}$ FV3000 Confocal Laser Scanning Microscope}

\section{Advance your research with the next evolution of confocal laser scanning microscope technology}

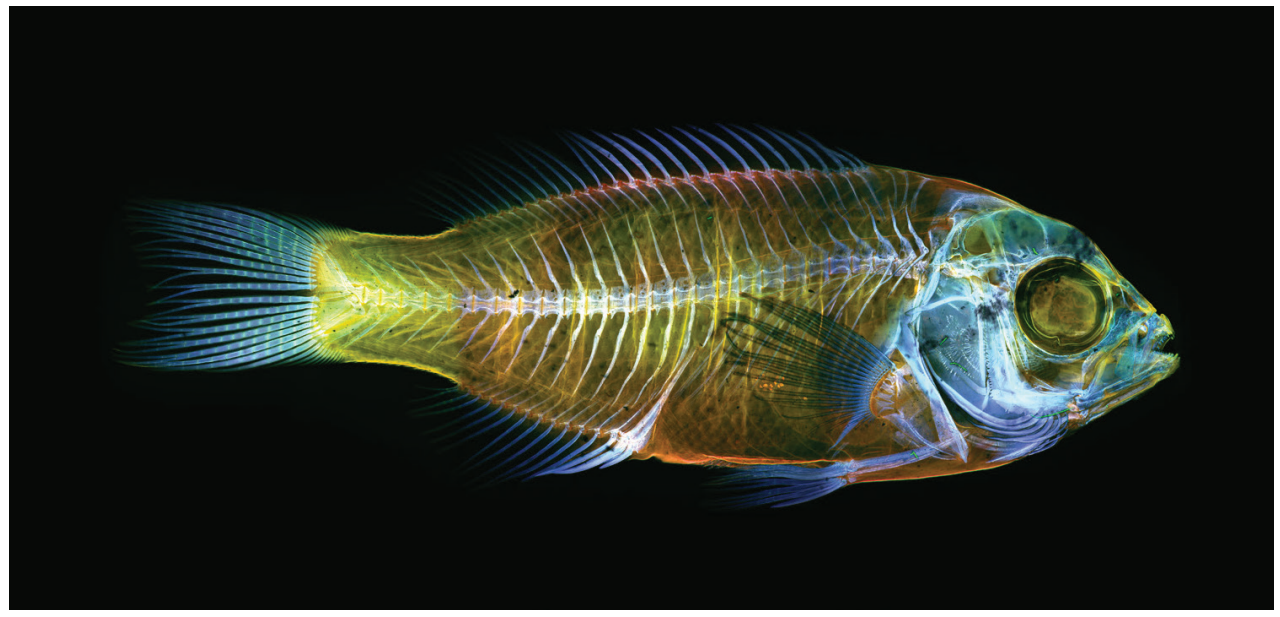

Captured using a 1.25x objective on the FV3000 system

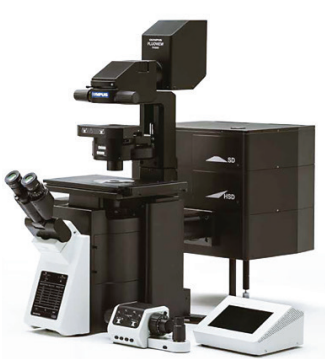

- Capture up to 438 frames per second

- Optimized for live cell imaging with highefficiency TruSpectral detection on every channel
- Designed for easy "macro to micro" imaging from $1.25 \times$ to $150 x$

- Fully upgradable modular design with up to 4 channels of high-sensitivity GaAsP detection

\section{Your Science Matters ${ }^{\mathrm{TM}}$}

